\title{
Chaotic social interaction via endogenous reactivity
}

\section{Ahmad Naimzada \& Marina Pireddu}

To cite this article: Ahmad Naimzada \& Marina Pireddu (2014) Chaotic social interaction via endogenous reactivity, Journal of Difference Equations and Applications, 20:8, 1222-1239, DOI: 10.1080/10236198.2014.903938

To link to this article: http://dx.doi.org/10.1080/10236198.2014.903938

曲 Published online: 08 May 2014.

Submit your article to this journal $₫$

III Article views: 42

Q View related articles $\sqsubset$

View Crossmark data $\asymp$

4 Citing articles: 1 View citing articles $2 \pi$ 


\title{
Chaotic social interaction via endogenous reactivity
}

\author{
Ahmad Naimzada ${ }^{\mathrm{a} 1}$ and Marina Pireddu ${ }^{\mathrm{b}} *$ \\ ${ }^{a}$ Department of Economics, Quantitative Methods and Management, University of Milano-Bicocca, \\ U7 Building, Via Bicocca degli Arcimboldi 8, 20126 Milan, Italy; ${ }^{b}$ Department of Mathematics and \\ Applications, University of Milano-Bicocca, U5 Building, Via Cozzi 55, 20125 Milan, Italy
}

(Received 24 October 2013; final version received 9 March 2014)

\begin{abstract}
We propose a framework to analyse the dynamical process of decision and opinion formation of two economic homogeneous and boundedly rational agents that interact and learn from each other over time. The decisional process described in our model is an adaptive adjustment mechanism in which two agents take into account the difference between their own opinion and the opinion of the other agent. The smaller that difference, the larger the weight given to the comparison of the opinions. We assume that if the distance between the two opinions is larger than a given threshold, then there is no interaction and the agents do not change their opinion anymore. Introducing an auxiliary variable describing the distance between the opinions, we obtain a one-dimensional map for which we investigate, mainly via analytical tools, the stability of the steady states, their bifurcations, as well as the existence of chaotic dynamics and multistability phenomena.
\end{abstract}

Keywords: adaptive decisional mechanism; bifurcations; multistability; complex dynamics

AMS Subject Classification: 37N40; 39A28; 39A60; 91B55

\section{Introduction}

In this paper we propose an approach according to which economic and social decisions are the result of social interactions of homogeneous and boundedly rational agents that learn from each other over time. In fact, differently from the traditional economic models, in our framework the dynamical process is not explained by optimal intertemporal trajectories that are defined by constant preferences and well-defined budget constraints, but rather by the behaviour of homogeneous interacting agents that adapt their decisions in accordance with the other players' choices. Our model conforms with some approaches analysing the motivations of decision-making within disciplines such as sociology, psychology and marketing [2]. In the present paper we do not consider what is called intrinsic utility of the decision process, that is, utility derived from the economic and social activities. We take into account only external influences derived from the observation of the others' behaviour: we adopt such an approach in order to highlight the role of social interaction as a source of continuous update of the decisional process.

More precisely, in the present paper we propose a framework with two interacting agents, in which each agent weights the decision or the opinion of the other agent to a certain extent in forming his/her own new decision or opinion. This process is modelled by an adaptive adjustment mechanism. In particular, similarly to what was assumed in $[5,14]$,

*Corresponding author. Email: marina.pireddu@unimib.it 
the weight with which an agent takes into account the difference between his/her own opinion and the opinion of the other agent is related to the distance between the two opinions, that is, the smaller that distance, the larger the weight given to the comparison of the opinions. However, differently from [5,14], we assume that if the distance between the two opinions is larger than a given threshold, then there is no interaction and each agent does not change his/her own opinion anymore.

Given this decisional mechanism, we tackle the issue of the evolution of the opinions over time, that is, whether they converge towards unanimity or whether they give rise to other kinds of dynamical behaviours. In doing this, we introduce an auxiliary variable, describing the distance between the two agents' opinions. In such a way, we are led to consider a onedimensional (1D) dynamical system, for which we find a steady state in the origin, corresponding to the unanimity scenario, and a continuum of steady states related to the situation in which opinions are much different and thus agents do not interact and do not modify their opinions anymore. From a dynamical point of view, the only interesting steady state is the origin, given the instability of all other steady states. We find that an excessive reactivity destabilizes the unanimity fixed point through a first period-doubling bifurcation. A further increase in the reactivity parameter destabilizes the period-two cycle that, differently from the classical period-doubling bifurcation scenario, gives rise, through a double pitchfork bifurcation of the second iterate, to two coexisting period-two cycles, which in turn bifurcate giving rise to a sequence of coexisting attractors of the same type until the emergence of chaos.

Among the results above, we analytically investigate the stability of the unanimity steady state and the flip bifurcation through which it loses the stability, the double pitchfork bifurcation of the second iterate and the presence of chaotic dynamics. In particular, the existence of chaos is proved via the expander method in [7]. For the reader's convenience, we recall which concepts that technique is based on and we compare it with other wellknown approaches in the chaos literature, explaining why, in the present framework, they are not convenient, or simply not working. In doing this, we also prove the existence of an absorbing interval for our dynamical system when the reactivity parameter is not too large.

On the other hand, due to the complexity of the computations involved, we show only numerically the subsequent period-doubling bifurcations of the coexisting periodic attractors leading to two coexisting periodic attractors, which merge into a unique attractor when increasing the reactivity parameter.

The remainder of the paper is organized as follows. In Section 2 we present our model. In Section 3 we perform the stability and bifurcation analysis, both analytically and numerically. In Section 4 we rigorously prove the presence of an absorbing interval and of complex dynamics, and we compare the technique we employ with other techniques in the existing chaos literature. In Section 5 we discuss our results and draw some conclusions.

\section{The model}

Consider two agents, $A$ and $B$, between whom a decision formation process takes place. We suppose that each agent takes into account the decision of the other agent to a certain extent in forming his/her new own decision. This dynamic adaptive decision process can be repeated again and again and leads to a dynamical process in discrete time. It is assumed that the decision of each agent is expressed by a real number, that is, the process is represented as a continuous decision dynamics, in contrast to the case of binary decision dynamics. For each agent $i \in\{A, B\}$, we denote his/her decision at time $t \in\{0,1,2, \ldots\}$ by $y_{i}(t)$. Thus, $y_{i}(t)$ is a real number and the two-dimensional (2D) vector $y(t)=$ $\left(y_{A}(t), y_{B}(t)\right) \in \mathbb{R}^{2}$ represents the decision profile at time $t$. Fixing an agent $i \in\{A, B\}$, the 
variation between his/her decisions at time $t+1$ and at time $t$ is proportional to the difference between his/her own decision and the decision of the other agent at time $t$ and to an endogenous nonnegative reactivity, we denote by $\gamma_{i}(t)$. With this notation, decision formation of agent $i$ can be described by the following adaptive scheme

$$
y_{i}(t+1)=y_{i}(t)+\gamma_{i}(t)\left(y_{j}(t)-y_{i}(t)\right), \quad i \neq j \in\{A, B\},
$$

where $\gamma_{i}(t)$ is an endogenous reactivity decreasing with the distance between the decisions of the two agents. More precisely, we assume that the agents are homogeneous in the reactivity, that is, $\gamma_{A}(t)=\gamma_{B}(t)=\gamma(t)$, with

$$
\gamma(t)= \begin{cases}\alpha-\beta\left|y_{A}(t)-y_{B}(t)\right|, & \text { for }\left|y_{A}(t)-y_{B}(t)\right| \leq \frac{\alpha}{\beta}, \\ 0, & \text { for }\left|y_{A}(t)-y_{B}(t)\right|>\frac{\alpha}{\beta}\end{cases}
$$

where $\alpha$ and $\beta$ are positive constants.

Inserting $\gamma(t)$ from (2.2) into (2.1), we obtain the 2D system

$$
\left\{\begin{array}{l}
y_{A}(t+1)=y_{A}(t)+\gamma(t)\left(y_{B}(t)-y_{A}(t)\right), \\
y_{B}(t+1)=y_{B}(t)+\gamma(t)\left(y_{A}(t)-y_{B}(t)\right) .
\end{array}\right.
$$

Introducing the auxiliary variable $z(t)=y_{A}(t)-y_{B}(t)$, representing the difference between the two decisions at time $t$, and subtracting the two equations in (2.3), we obtain

$$
z(t+1)= \begin{cases}(1-2 \alpha) z(t)-2 \beta z(t)^{2}, & \text { for }-\frac{\alpha}{\beta} \leq z(t)<0, \\ (1-2 \alpha) z(t)+2 \beta z(t)^{2}, & \text { for } \frac{\alpha}{\beta} \geq z(t) \geq 0 \\ z(t), & \text { else }\end{cases}
$$

Through the change of variable ${ }^{2} x(t)=-(2 \beta /(1-2 \alpha)) z(t)$, we can rewrite (2.4) as follows

$$
x(t+1)= \begin{cases}(1-2 \alpha)\left(x(t)+x(t)^{2}\right), & \text { for } \frac{2 \alpha}{1-2 \alpha} \leq x(t)<0, \\ (1-2 \alpha)\left(x(t)-x(t)^{2}\right), & \text { for }-\frac{2 \alpha}{1-2 \alpha} \geq x(t) \geq 0, \\ x(t), & \text { else. }\end{cases}
$$

Setting now $\mu=-(1-2 \alpha)$, we finally obtain

$$
x(t+1)= \begin{cases}-\mu\left(x(t)+x(t)^{2}\right), & \text { for }-\frac{\mu+1}{\mu} \leq x(t)<0 \\ -\mu\left(x(t)-x(t)^{2}\right), & \text { for } \frac{\mu+1}{\mu} \geq x(t) \geq 0 \\ x(t), & \text { else }\end{cases}
$$


with $\mu$ positive parameter (since $\alpha>1 / 2$ ), whose influence we will investigate in the next sections. Indeed, the dynamic equation in (2.6) is the one we are going to consider in the remainder of the paper because, differently from the $2 \mathrm{D}$ formulation in (2.3), it can be studied analytically and still allows to get results that are easily interpretable. For instance, as we shall see in Section 3, the unique dynamically interesting steady state is the origin, which coincides with the conformism scenario.

In view of the subsequent analysis, it is expedient to consider the $1 \mathrm{D}$ map $f: \mathbb{R} \rightarrow \mathbb{R}$ related to (2.6), defined as

$$
f(x ; \mu)= \begin{cases}-\mu\left(x+x^{2}\right), & \text { for } \quad-\frac{\mu+1}{\mu} \leq x<0, \\ -\mu\left(x-x^{2}\right), & \text { for } \frac{\mu+1}{\mu} \geq x \geq 0, \\ x, & \text { else. }\end{cases}
$$

When comparing the dynamical system generated by the reactivity in (2.2) with the one analysed in [14], some crucial differences emerge. Indeed, although the striking similarity between the shape of the graph of the maps generating the two dynamical systems, we stress that map $f$ in (2.7) permits an analytical treatment, we are going to perform in the next sections, while the map considered in [14] is studied mainly numerically, because of the complexity of the computations involved. Moreover, as observed in Section 1, also the decisional mechanisms leading to their formulations differ. In fact, in the present framework, the reactivity in (2.2) vanishes when the distance between the two opinions is large enough, while the reactivity considered in [14] is decreasing with the distance between the two opinions and tends to zero in the limit, but it never vanishes.

\section{Stability and bifurcation analysis}

As a first step in the analysis of our system, in the next result we study the stability conditions for the fixed point $x=0$. There exists in fact also the continuum of symmetric fixed points $\{ \pm x: x \in[(\mu+1) / \mu,+\infty)\}$, but they are not locally asymptotically stable and thus not interesting in view of the subsequent bifurcation analysis.

Proposition 3.1. For map $f$ in (2.7) the fixed point $x=0$ is locally asymptotically stable if $\mu<1$.

Proof. The conclusion immediately follows by observing that for every $x$ in a sufficiently small neighbourhood of 0 , it holds ${ }^{3}$ that

$$
\frac{\partial f}{\partial x}(x ; \mu)=\left\{\begin{array}{cl}
-2 \mu x-\mu, & \text { for } x \leq 0, \\
2 \mu x-\mu, & \text { for } x \geq 0 .
\end{array} .\right.
$$

Thus $(\partial f / \partial x)(0 ; \mu)=-\mu \in(-1,1)$ if and only if $(0<) \mu<1$.

Hence, for $\mu<1$, the origin is a stable fixed point and it loses its stability for $\mu$ just above 1. In Lemma 3.2 below we show that the stability is lost via a period-doubling 
bifurcation. In the proof we will use ([6], Theorem 2.7, p. 79) which, like other results we will need hereinafter, requires to work with the second iterate of map $f$. We notice that the formulation of $f^{2}$ slightly changes according to the value of $\mu$. Indeed, if the maximum value of $f$ does not exceed the value of the fixed point $(\mu+1) / \mu$ (and, symmetrically, the minimum value of $f$ is above the value of the fixed point $-(\mu+1) / \mu)$, which, as shown in Proposition 4.2, happens for $\mu<2+\sqrt{8}$, then there exists an absorbing interval and the expression of $f^{2}$ gets slightly simplified as follows:

$$
f^{2}(x ; \mu)= \begin{cases}\mu^{2} x(1+x)(1-\mu x(x+1)), & \text { for } x \in\left[-\frac{\mu+1}{\mu},-1\right], \\ \mu^{2} x(1+x)(1+\mu x(x+1)), & \text { for } x \in[-1,0], \\ \mu^{2} x(1-x)(1+\mu x(x-1)), & \text { for } x \in[0,1], \\ \mu^{2} x(x-1)(\mu x(x-1)-1), & \text { for } x \in\left[1, \frac{\mu+1}{\mu}\right], \\ x, & \text { else. }\end{cases}
$$

Since in the results below we will consider $1+\sqrt{6}$ as largest value for $\mu$, this is the only formulation of $f^{2}$ we will use.

LeMma 3.2. For map $f$ in (2.7) a period-doubling bifurcation occurs at $x=0$ when $\mu=1$.

Proof. In view of ([6], Theorem 2.7, p. 79), we just have to check the following conditions:

(C1) $f(0 ; \mu)=0$, for all $\mu$ in a neighbourhood of 1 ;

(C2) $\frac{\partial f}{\partial x}(0 ; 1)=-1$;

(C3) $\frac{\partial^{2} f^{2}}{\partial x \partial \mu}(0 ; 1) \neq 0$.

Condition $(\mathrm{C} 1)$ is trivially satisfied since $f(0 ; \mu)=0$, for all $\mu$.

As regards $(\mathrm{C} 2)$, notice that $(\partial f / \partial x)(0 ; \mu)=-\mu$. Hence $(\partial f / \partial x)(0 ; 1)=-1$, as desired.

In order to check (C3), a direct computation starting from (3.1) shows that $\left(\partial^{2} f^{2} / \partial x \partial \mu\right)(0 ; \mu)=2 \mu$. Thus $\left(\partial^{2} f^{2} / \partial x \partial \mu\right)(0 ; 1)=2 \neq 0$. This concludes the proof.

In order to understand when new points of period two get born in the region $[-1,1]$, that is, the interesting area from a dynamical viewpoint, ${ }^{4}$ let us consider $\left.f^{2}\right|_{[-1,1]}$. In fact, since map $f$ is odd, and thus $f^{2}$ is odd, too, we will restrict our attention to $\left.f^{2}\right|_{[0,1]}$. The conclusions drawn there will then hold for $\left.f^{2}\right|_{[-1,0]}$ as well, just changing sign where needed.

The next result gathers some information about the period-two points on $[0,1]$ and their stability.

Proposition 3.3. In addition to the fixed point $x=0$, the points of period two for $f$ on $[0,1]$ are:

- for $\mu>1, \tilde{x}=(\mu-1) / \mu$; 
- for $\mu>3, \quad \bar{x}=\left((\mu+1)-\sqrt{\mu^{2}-2 \mu-3}\right) /(2 \mu) \quad$ and $\quad \overline{\bar{x}}=\quad((\mu+1)+$ $\left.\sqrt{\mu^{2}-2 \mu-3}\right) /(2 \mu)$.

In particular, $\tilde{x}$ gets originated when the origin undergoes a period-doubling bifurcation for $\mu=1$ and $\tilde{x}$ is locally asymptotically stable for $1<\mu<3$. For $\mu=3$, $\tilde{x}$ undergoes $a$ pitchfork bifurcation and $\bar{x}$ and $\overline{\bar{x}}$ get born, which are locally asymptotically stable for $3<\mu<1+\sqrt{6}$.

Proof. The points of period two $\tilde{x}, \bar{x}$ and $\overline{\bar{x}}$ are found by solving the equation $\left.f^{2}(x ; \mu)\right|_{[0,1]}=\mu^{2} x(1-x)(1+\mu x(x-1))=x \in[0,1]$. As usual, the stability conditions are determined by setting $\left(\partial f^{2} / \partial x\right)(\hat{x} ; \mu) \in(-1,1)$, where $\hat{x}$ is one of the periodic points found above. The period-doubling bifurcation of the origin has been proven in Lemma 3.2, while, according to [21], the pitchfork bifurcation of $\tilde{x}$ can be shown by checking the following conditions:

$$
\begin{aligned}
\left(\mathrm{C}^{\prime}\right) \quad f^{2}(\tilde{x} ; 3) & =\tilde{x} ; \\
\left(\mathrm{C} 2^{\prime}\right) \quad \frac{\partial f^{2}}{\partial x}(\tilde{x} ; 3) & =1 ; \\
\left(\mathrm{C}^{\prime}\right) \quad \frac{\partial f^{2}}{\partial \mu}(\tilde{x} ; 3) & =0, \quad \frac{\partial^{2} f^{2}}{\partial x^{2}}(\tilde{x} ; 3)=0, \\
\frac{\partial^{2} f^{2}}{\partial x \partial \mu}(\tilde{x} ; 3) & \neq 0, \quad \frac{\partial^{3} f^{2}}{\partial x^{3}}(\tilde{x} ; 3) \neq 0 .
\end{aligned}
$$

As regards $\left(\mathrm{C}^{\prime}\right)$, a direct computation shows that $f^{2}(\tilde{x} ; \mu)=\tilde{x}$, for every $\mu>1$. With regard to $\left(\mathrm{C} 2^{\prime}\right)$, it is easy to prove that, for $\mu>1,\left(\partial f^{2} / \partial x\right)(\tilde{x} ; \mu)=1$ if and only if $\mu=3$. As concerns $\left(\mathrm{C}^{\prime}\right)$, it holds that, for $\mu>1,\left(\partial f^{2} / \partial \mu\right)(\tilde{x} ; \mu)=((3-\mu)(\mu-1)) / \mu^{2}=0$ if and only if $\mu=3$. Moreover, $\left(\partial^{2} f^{2} / \partial x^{2}\right)(\tilde{x} ; \mu)=2 \mu\left(-\mu^{2}+5 \mu-6\right)=0$ for $\mu=3$ and $\left(\partial^{2} f^{2} / \partial x \partial \mu\right)(\tilde{x} ; 3)=2 \neq 0$. Finally, $\left(\partial^{3} f^{2} / \partial x^{3}\right)(\tilde{x} ; 3)=-108 \neq 0$. This concludes the proof.

With regard to the points of period two on $[0,1]$ found in Proposition 3.3 and their symmetric counterpart on $[-1,0]$, it is easy to check that

$$
\begin{aligned}
f\left(\frac{\mu-1}{\mu} ; \mu\right) & =\frac{1-\mu}{\mu}, \\
f\left(\frac{(\mu+1)-\sqrt{\mu^{2}-2 \mu-3}}{2 \mu} ; \mu\right) & =\frac{-(\mu+1)-\sqrt{\mu^{2}-2 \mu-3}}{2 \mu}
\end{aligned}
$$

and

$$
f\left(\frac{(\mu+1)+\sqrt{\mu^{2}-2 \mu-3}}{2 \mu} ; \mu\right)=\frac{-(\mu+1)+\sqrt{\mu^{2}-2 \mu-3}}{2 \mu} .
$$

From the previous results, it looks evident that an increasing value for $\mu$ makes the behaviour of map $f$ more and more complex, with the loss of stability of the previously existing periodic points and the emergence of new periodic orbits. We illustrate this 
(A)



(B)



Figure 1. The graph of $f$ with $\mu=0.5$ in (A) and $\mu=2$ in (B), respectively.

phenomenon in Figures 1 and 2. In particular, in Figure 1(A) parameter $\mu$ is set equal to 0.5 and thus, according to Proposition 3.1, the unique fixed point, i.e. the origin, is locally stable. In Figures 1(B) and 2(A), where we set $\mu=2$, according to Lemma 3.2, the origin has lost its stability via a flip bifurcation and, according to Proposition 3.3, the points of period two $\tilde{x}=(1 / 2)$ and its symmetric counterpart $-\tilde{x}$ have emerged. In Figure 2(B) we increase parameter $\mu$ to 3.3 , so that, according to Proposition 3.3, the two periodic points $\pm \tilde{x}=$ $\pm 0 . \overline{69}$ lose their stability via a double pitchfork bifurcation and the period-two points $\bar{x}=0.479$ and $\overline{\bar{x}}=0.823$, as well as their symmetric counterparts $-\bar{x}$ and $-\overline{\bar{x}}$, emerge. In Figure 2(C) we set $\mu=3.7>1+\sqrt{6}$ and thus, according to Proposition 3.3, also $\pm \bar{x}=$ \pm 0.39 and $\pm \overline{\bar{x}}= \pm 0.88$ are no more stable.

When comparing the process described above with what happens to the logistic map when increasing the corresponding parameter, in addition to several similarities, we also remark a crucial difference. In fact, instead of undergoing the standard period-doubling route to chaos characteristic of the logistic map, we observe for map $f$ in (2.7) that when the first orbit of period two becomes unstable, two new cycles of period two, coexisting with the 
(A)

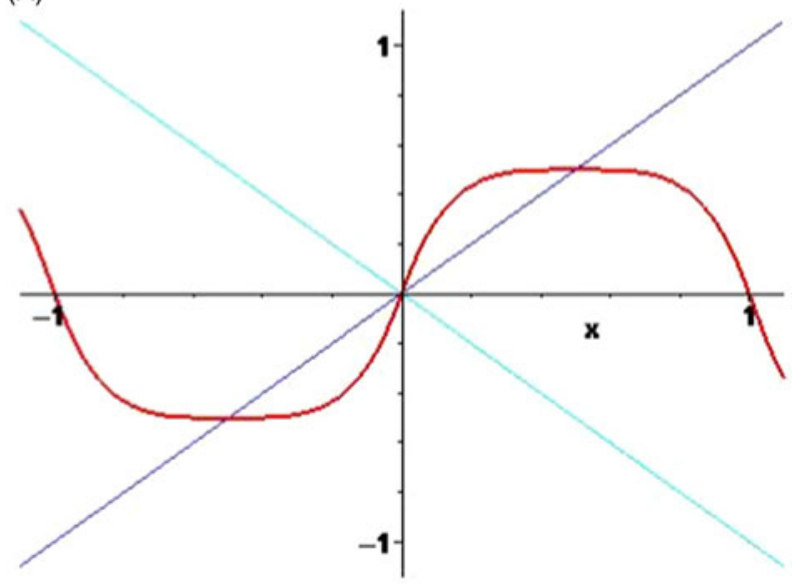

(B)

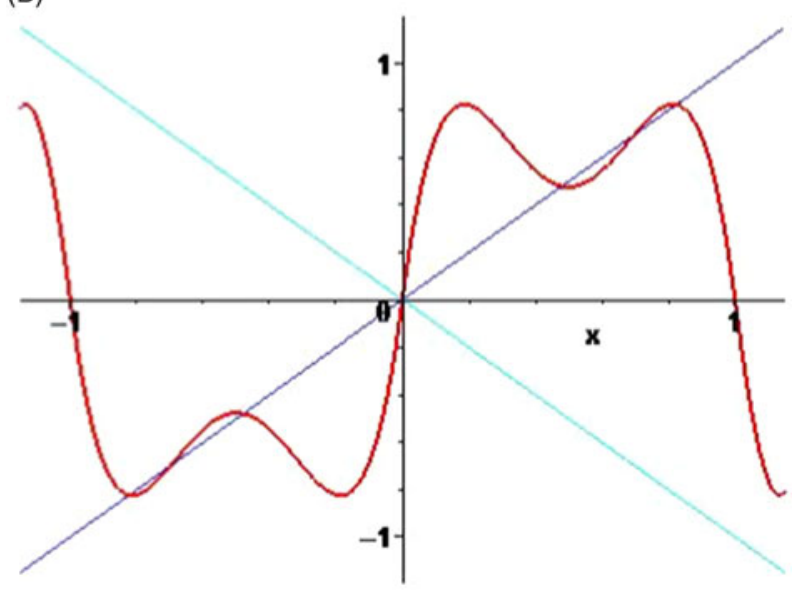

(C)

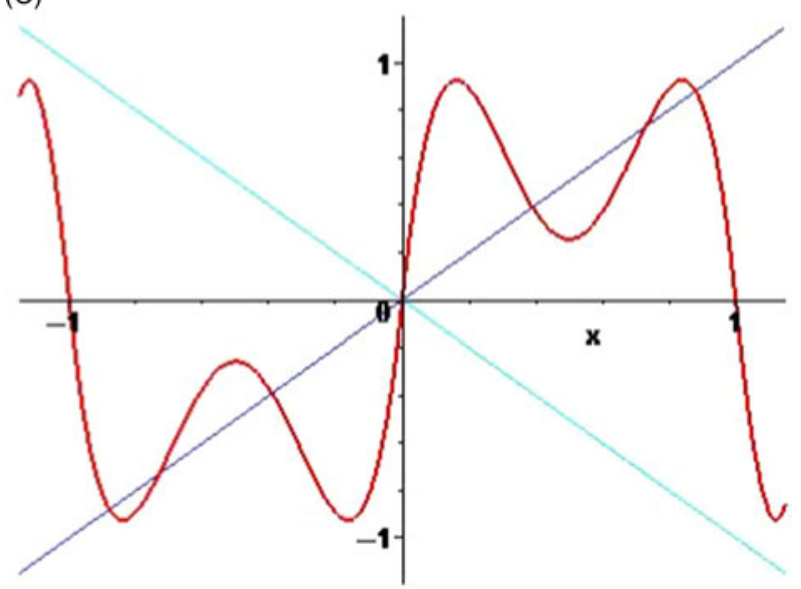

Figure 2. The graph of $f^{2}$ with $\mu=2$ in (A), $\mu=3.3$ in (B) and $\mu=3.7$ in (C), respectively. 


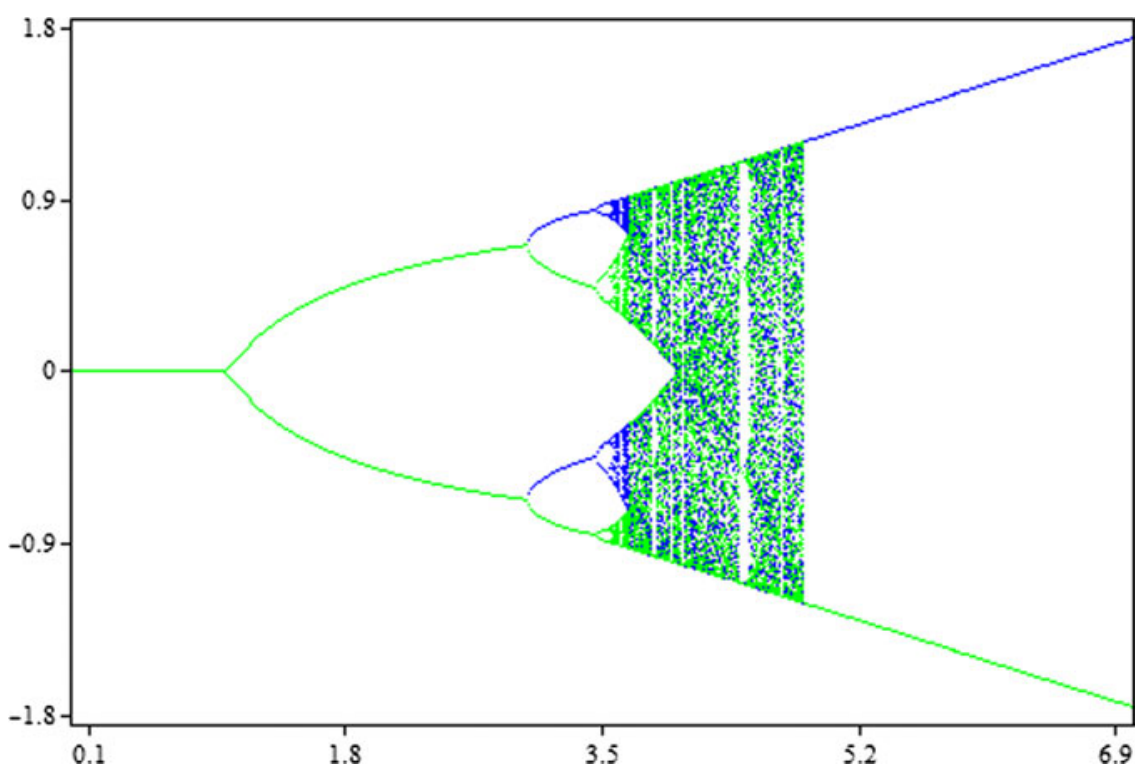

Figure 3. (Colour online) The bifurcation diagram for $f$ w.r.t. $\mu \in(0,7]$. Source: The blue dots refer to the initial condition $x(0)=-0.5$, while the green dots are obtained for the initial condition $x(0)=0.5$.

old one, emerge through a double pitchfork bifurcation of $f^{2}$. Such new cycles are stable at the beginning, but increasing $\mu$ they lose stability via period-doubling bifurcations leading to two coexisting cycles of period four, which again become unstable for larger values of $\mu$.

Due to the difficulty in computing the forth iterate of map $f$, we omit the analytical treatment of the latter steps and in the next section we directly prove the existence of chaotic dynamics. For the reader's convenience, we however present in Figure 3 the bifurcation diagram of $f$ with respect to $\mu$, which illustrates the steps above and also shows that the chaotic regime is interrupted by some periodicity windows. We stress that there is a perfect agreement between the theoretical results and the first bifurcation values in Figure 3. In particular, we observe the pitchfork bifurcation of the period-two cycle when $\mu=3$ and the subsequent emergence of two coexisting period-two cycles, which in turn bifurcate when $\mu=1+\sqrt{6} \approx 3.45$ giving rise to two coexisting period-four cycles. The latter bifurcate for $\mu \approx 3.545$ generating two coexisting period-eight cycles, which in turn give rise to two coexisting chaotic attractors in eight pieces when $\mu \approx 3.574$, generating two coexisting chaotic attractors in four pieces when $\mu \approx 3.576$. Those give rise to two coexisting chaotic attractors in two pieces when $\mu \approx 3.593$, which generate a unique chaotic attractor in two pieces when $\mu \approx 3.673$, which in turn generates a unique chaotic attractor in one piece when $\mu=4$. A periodicity window can be observed for instance for $\mu \approx 3.82$. As we shall see in Proposition 4.2 below, for $\mu<2+\sqrt{8}$, there exists an absorbing interval $I \subset(-((\mu+1) / \mu),(\mu+1) / \mu)$. When however $\mu$ exceeds that threshold, then, for any initial condition, the iterates of map $f$ will eventually hit the set $(-\infty,-((\mu+1) / \mu)] \cup$ $[(\mu+1) / \mu,+\infty)$, entirely composed of fixed points. This is the reason why in Figure 3 for $\mu>2+\sqrt{8} \approx 4.828$ the chaotic band ends and two segments appear in its place.

\section{Chaotic dynamics}

In order to prove the presence of chaotic dynamics for our system, we apply the expander method from [7]. More specifically, in Proposition 4.1 below we will use the Chaos 
Lemma in [7]. With this respect, we recall hereinafter some useful definitions. We warn the reader that sometimes we will replace the general setting in [7] with the specific framework we are considering.

Let $Q$ be a metric space, $Q_{0}$ be a compact subset of $Q$ and $\phi: Q_{0} \rightarrow Q$ be a continuous map. Let $S_{1}$ and $S_{2}$ be non-empty, compact and disjoint subsets of $Q_{0}$ and let $\mathcal{E}$ be a nonempty family of non-empty subsets of $Q$ such that, for each $E \in \mathcal{E}$ and each $S_{i}, i \in\{1,2\}$, there exists a compact set $P_{i} \subset E \cap S_{i}$ such that $\phi\left(P_{i}\right) \in \mathcal{E}$. The family $\mathcal{E}$ is called family of expanders in [7] and each $E \in \mathcal{E}$ is called expander.

The Chaos Lemma (cf. [7], Lemma 4) states that if the assumptions above are fulfilled, then there exists a compact, invariant set $Q_{*} \subset Q_{0}$ such that map $\phi$ displays on it sensitive dependence with respect to initial conditions and each sequence on two symbols is followed by the $\phi$ orbit of a point in $Q_{*}$.

Before going on with the description and the application of the results in [7], let us illustrate through some pictures what is the idea behind the definitions above. The expander method is in fact related to the literature on 'topological horseshoes' (see, for instance $[4,13,19,22]$ ), which has as starting point the celebrated construction by Smale [18]. The problem with the original Smale horseshoe is that in the applications it requires to verify suitable differentiability and hyperbolicity conditions, which either are not fulfilled or are hard to check for a given map. Hence, more general and less stringent definitions of horseshoe have been suggested in order to reproduce some geometrical features typical of the Smale horseshoe while discarding the hyperbolicity conditions.

Since the original horseshoe map is $2 \mathrm{D}$, let us start with a planar illustration of the expander method, explaining then how it looks like in the 1D context we are going to consider in our application. In fact, in Figure 4 we represent a rotated version of the original Smale horseshoe. ${ }^{5}$

In this case we enter the framework in [7] with the following positions: $Q$ is the Euclidean plane, $Q_{0}$ is the unit square and $\phi:[0,1]^{2} \rightarrow \mathbb{R}^{2}$ is the 'rotated Smale horseshoe map', which acts on the unit square as shown in Figure 4. The square is first shrunk uniformly along the vertical direction and expanded along the horizontal one. Subsequently, the elongated rectangle obtained in the previous step is bent in order to cross the original square twice. The resulting map $\phi$ is a diffeomorphism transforming the horizontal lines of the square into lines crossing twice the domain horizontally. For instance, in Figure 4 we show how the horizontal rectangle $E$ is transformed by $\phi$. In particular, we depicted with a darker colour the sub-rectangles $P_{1}$ and $P_{2}-$ as well as their image-set - obtained intersecting the horizontal rectangle $E$ with the vertical rectangles $S_{1}$ and $S_{2}$, respectively. Notice that $\phi\left(P_{1}\right)$ and $\phi\left(P_{2}\right)$ are two rectangles crossing the square horizontally, like $E$ does. Hence, by the genericity of $E$, we can conclude that, for the above choice of $S_{1}$ and $S_{2}$, every rectangle (more generally, every compact and connected set) joining the vertical sides of the square, and thus crossing

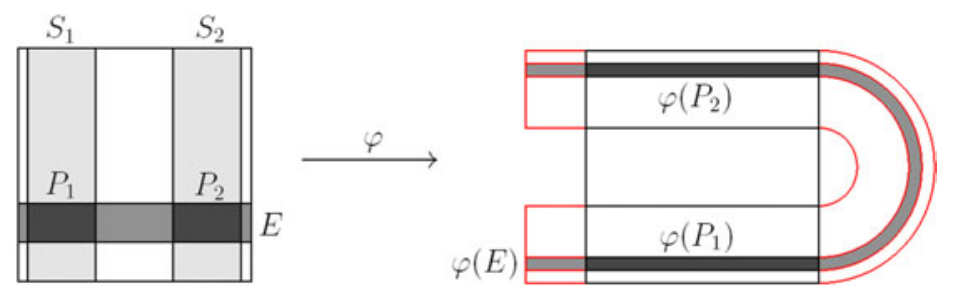

Figure 4. A graphical illustration of how the 'rotated Smale horseshoe map' acts on the unit square. 
the latter horizontally, is an expander, ${ }^{6}$ as the $\phi$ images of its intersections with $S_{1}$ and $S_{2}$ have the same property.

Let us now illustrate how the concepts in [7] work in the 1D context. To such aim, we will consider the well-known logistic map restricted to $[0,1]$, that is $\phi:[0,1] \rightarrow \mathbb{R}, x \mapsto \mu x(1-x)$, so that $Q$ is the real line and $Q_{0}$ is the unit interval. It is easy to see that, as long as $\mu>4$, the maximum value of $\phi$ exceeds 1 . Calling then

$$
x_{1}:=\frac{1-\sqrt{1-(4 / \mu)}}{2}, \quad x_{2}:=\frac{1+\sqrt{1-(4 / \mu)}}{2}
$$

the two solutions to the equation $\phi(x)=1$ for $\mu>4$, we set $S_{1}=\left[0, x_{1}\right], S_{2}=\left[x_{2}, 1\right]$ and we claim that $E=[0,1]$ is an expander. Indeed, defining $P_{i}=E \cap S_{i}=S_{i}, i \in\{1,2\}$, it holds that $\phi\left(P_{1}\right)=\phi\left(P_{2}\right)=[0,1]=E$ (see Figure 5). Actually, $E=[0,1]$ is the only possible (connected) expander. In fact, the latter framework may be seen as a 'flattened' version of the $2 \mathrm{D}$ context exposed above, in which the length of the vertical sides of the unit square tends to zero and the role played in the previous example by horizontal rectangles can now be played just by the unit interval. In order to pursue further the comparison with the Smale horseshoe, we finally notice that in the 1D framework the image-set is placed on the $y$-axis but, to have a better analogue of the planar setting, it should be rather imagined on the $x$-axis.

Turning back to the description of the results in [7] and, in particular, to the Chaos Lemma recalled above, we stress that, from the proof given in [7], beyond the properties of the compact, invariant set $Q_{*} \subset Q_{0}$ stated in ([7], Lemma 4) (that is map $\phi$ displays on $Q_{*}$ sensitive dependence with respect to initial conditions and each sequence on two symbols is followed by the $\phi$ orbit of a point in $Q_{*}$ ), it also follows that map $\phi$ is topologically

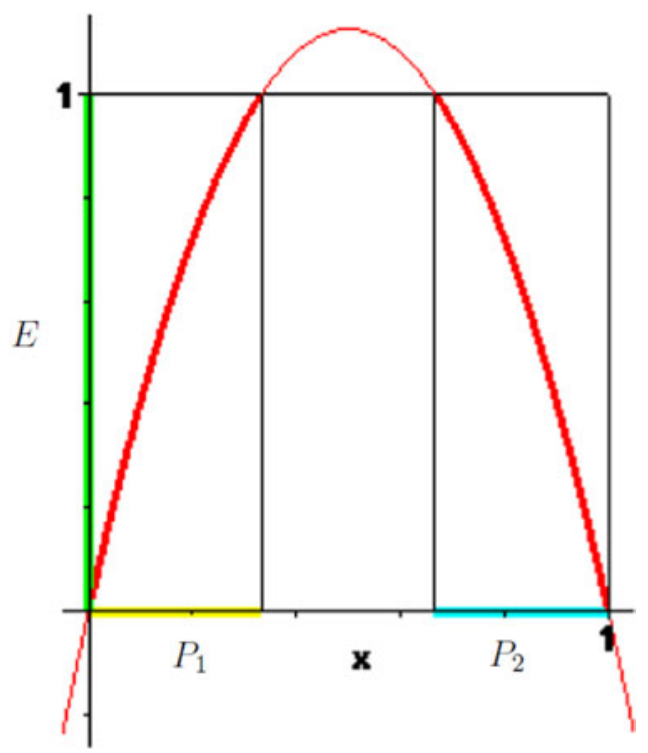

Figure 5. (Colour online) The graph of the logistic map with $\mu=4$.5. Source: The yellow and cyan sets on the $x$-axis are $P_{1}=S_{1}$ and $P_{2}=S_{2}$, respectively, and the green interval on the $y$-axis is their image-set $E=[0,1]$. 
transitive on $Q_{*}$. In order to explain the latter step, we need to recall some further definitions. We name dynamical systems the pairs $(Z, l)$, where $Z$ is a compact metric space and $l: Z \rightarrow Z$ is continuous and onto. Given $z \in Z$, we call the set $\gamma(z):=\left\{l^{n}(z): n \in \mathbb{N}\right\}$ the forward $l$ orbit of $z$ and we denote by $\overline{\gamma(z)}$ its closure. Given $z \in Z$, we define the $\omega$-limit set of $z$ as $\omega(z):=\left\{x \in Z: \exists n_{j} \nearrow+\infty\right.$ with $\left.l^{n_{j}}(z) \rightarrow x\right\}$. According to ([20], Theorem 5.5), $\omega(z)$ is closed, non-empty and invariant by the compactness of $Z$. Moreover it holds that $\overline{\gamma(z)}=\gamma(z) \cup \omega(z)$.

Turning back to the proof of ([7], Lemma 4), therein it is shown that there exists $x^{*} \in Q_{0}$ such that $x^{*} \in \omega\left(x^{*}\right)$ and, by construction, $Q_{*}=\omega\left(x^{*}\right)$. Since $x^{*} \in \omega\left(x^{*}\right)$, by the invariance of the $\omega$-limit sets, also all iterates of $x^{*}$ belong to $\omega\left(x^{*}\right)$, and thus $\gamma\left(x^{*}\right) t \omega\left(x^{*}\right)$. Hence $\overline{\gamma\left(x^{*}\right)}=\gamma\left(x^{*}\right) \cup \omega\left(x^{*}\right)=\omega\left(x^{*}\right)=Q_{*}$ and then the forward $\phi$ orbit of $x^{*}$ is dense in $Q_{*}$. On the other hand, by the invariance of $Q_{*}$, the pair $\left(Q_{*},\left.\phi\right|_{Q_{*}}\right)$ is a dynamical system and, according to ([1], Lemma 3), in every dynamical system the existence of a dense orbit is equivalent to the topological transitivity of the associated map.

In order to apply the Chaos Lemma to prove the presence of a chaotic set in our context, in what follows we will consider $\mu>4$ and take ${ }^{7}$

$$
\begin{gathered}
Q=\mathbb{R}, \quad Q_{0}=[-1,1], \quad \phi=\left.f\right|_{[-1,1]}, \\
S_{1}=\left[-1, \frac{-\mu-\sqrt{\mu^{2}-4 \mu}}{2 \mu}\right] \cup\left[\frac{\mu+\sqrt{\mu^{2}-4 \mu}}{2 \mu}, 1\right], \\
S_{2}=\left[\frac{-\mu+\sqrt{\mu^{2}-4 \mu}}{2 \mu}, \frac{\mu-\sqrt{\mu^{2}-4 \mu}}{2 \mu}\right], \\
\mathcal{E}=\{E\}=\{[-1,1]\}, \quad P_{i}=E \cap S_{i}=S_{i}, \quad i \in\{1,2\} .
\end{gathered}
$$

See Figure 6 for a graphical illustration of the sets $S_{1}$ and $S_{2}$ when $\mu=4.5$.

Observe that, with the choices in (4.1)-(4.4), it holds that $\phi\left(P_{i}\right)=E, i \in\{1,2\}$, and thus the Chaos Lemma in [7] allows us to infer the existence of a chaotic set in $[-1,1]$. We summarize our findings in the next result.

Proposition 4.1. Let $\mu>4$. Then there exists a compact, invariant set $I^{*} \subset[-1,1]$ such that map $f$ displays on it sensitive dependence with respect to initial conditions, topological transitivity and each sequence on two symbols is followed by the forbit of a point in $I^{*}$.

We stress that we chose to employ the expander method in [7] because in the present framework the other approaches in the chaos literature we know do not allow to prove the presence of a complex behaviour or would lead to cumbersome computations. For instance, an attempt would be that of trying to apply the concept of covering intervals in [3] plus some tools from the theory of symbolic dynamics in $[9,11]$, in order to show that map $f$ has positive topological entropy, i.e. one of the trademarks of chaos. Another attempt would consist in using the 'stretching along the paths' (from now on, SAP) method, already employed, for instance, in $[12,17]$ to prove the existence of chaotic 


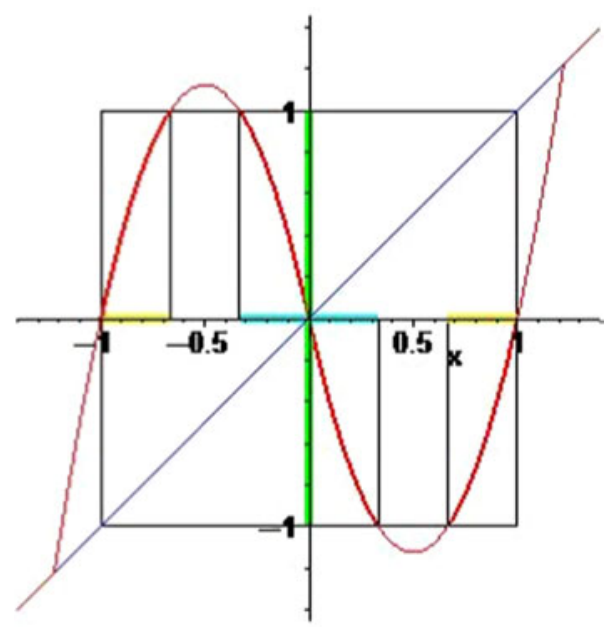

Figure 6. (Colour online) A graphical illustration of the construction behind Proposition 4.1 for $f$ with $\mu=4.5$. Source: In particular, the yellow and cyan sets on the $x$-axis are $S_{1}$ and $S_{2}$ in (4.2) and (4.3), respectively.

dynamics for both discrete and continuous-time dynamical systems. A third approach would consist in working with the first three iterates of map $f$ in (2.7), in order to find suitable intervals where to apply Theorem 1 in [10] to prove the existence of chaos in the sense of Li-Yorke, as described in conditions (T1) and (T2) in that result. This method has recently been used to rigorously prove the presence of chaotic dynamics in [14] (see [14], Proposition 4.2). Due to the similarity between the geometry of the present framework and the one in [14] we strongly believe that technique should work in the present framework as well. However, it would require to work even with the third iterate of map $f$, whose expression here is not quick to compute and easy to handle. For this reason we found more convenient to rather apply the expander method in [7].

Let us now turn back to the covering interval approach and the SAP method, in order to explain why they seem not applicable in our framework.

As concerns the covering interval approach, we recall that, given a continuous mapping $g: \mathbb{R} \rightarrow \mathbb{R}$ and two intervals $I, J \subseteq \mathbb{R}$, we say that $I g$ covers $J$ if $g(I) \supseteq J$, or equivalently, if there exists a sub-interval $I_{0} \subseteq I$ such that $g\left(I_{0}\right)=J$. Moreover, given a continuous onto map $g: I \rightarrow I$ defined on a compact interval $I \subset \mathbb{R}$ and $n \geq 2$ closed subintervals $I_{0}, \ldots, I_{n-1} \subseteq I$, with pairwise disjoint interiors, we associate with the dynamical system $(I, g)$ the $n \times n$ transition matrix $\mathbf{T}=\mathbf{T}(j, k)$, for $j, k \in\{0, \ldots, n-1\}$, defined as

$$
\mathbf{T}(j, k)= \begin{cases}1 & \text { if } I_{j} g \text { covers } I_{k} \\ 0 & \text { else. }\end{cases}
$$

It is possible to prove (cf. [9], Observation 1.4.2) that, when $\mathbf{T}$ is irreducible, i.e. for every pair of integers $j, k \in\{0, \ldots, n-1\}$ there exists a positive integer $\ell$ such that $\mathbf{T}^{\ell}(j, k)>0$, then $h_{\text {top }}(g) \geq \log (\bar{\lambda})$, where $\bar{\lambda}$ is the largest real eigenvalue of $\mathbf{T}$ in absolute value, also called Perron eigenvalue of $\mathbf{T}$.

As a first step towards the application of the just explained strategy, we need to find a compact invariant interval for our map $f$ in (2.7). In the next result we prove that, under 
certain conditions on parameter $\mu$, there exists an absorbing interval, which in particular is invariant for $\mu \geq 2 .^{8}$

Proposition 4.2. Call $m=1 / 2$ and $M=-1 / 2$ the unique local minimum point and local maximum point of map $f$, respectively, and set $m^{\prime}:=f(m)$ and $M^{\prime}:=f(M)$. If $\mu<2+\sqrt{8}$, the compact interval $I=\left[\mathrm{m}^{\prime}, M^{\prime}\right]$ is absorbing, i.e. there exists an open interval $J \supset I$ such that, for all $\bar{x} \in J$, there exists $\bar{n} \in \mathbb{N}$ such that $f^{\bar{n}}(\bar{x}) \in I$ and for any $x \in I, f^{n}(x) \in I$, for all $n \in \mathbb{N}$.

Proof. We claim that interval $J$ has as extreme points the fixed points $P_{1}=-((\mu+1) / \mu)$ and $P_{2}=(\mu+1) / \mu$, respectively.

Let us show at first that $J \supset I$. A simple calculation shows that since $\mu<2+\sqrt{8}$, then

$$
P_{1}=-\frac{\mu+1}{\mu}<m^{\prime}=-\frac{\mu}{4}<M^{\prime}=\frac{\mu}{4}<\frac{\mu+1}{\mu}=P_{2},
$$

as desired.

Let us then consider a generic starting point $\hat{x}$ in $J$ and show that its trajectory will eventually remain in $I$.

If $\hat{x} \in I$, by definition, $m^{\prime}=f(m) \leq f(\hat{x}) \leq f(M)=M^{\prime}$ and thus $f(\hat{x}) \in I$, as desired. Let us now analyse the two remaining cases, i.e. $P_{1}<\hat{x}<m^{\prime}$ and $P_{2}>\hat{x}>M^{\prime}$. Since $0 \in I$ and by continuity $f(x)>x$, for every $P_{1}<x<0$, and $f(x)<x$, for every $0<x<P_{2}$, if $P_{1}<\hat{x}<m^{\prime}$, then its iterates will approach $I$ in a strictly increasing way, while if $P_{2}>\hat{x}>M^{\prime}$, then its iterates will approach $I$ in a strictly decreasing way. Once that a forward iterate of $\hat{x}$ lies in $I$, then by construction all its subsequent iterates will be trapped inside $I$, as well. This concludes the proof.

A graphical illustration of the absorbing interval $I$ can be found in Figure 7 for $f$ with $\mu=4.5<2+\sqrt{8}$.



Figure 7. The highlighted set on the $x$-axis is the absorbing interval $I$ from Proposition 4.2. 
Notice that the threshold for the existence of an absorbing interval is higher than the threshold found in Proposition 4.1 for the existence of chaos. Thus, when $\mu \in(4,2+\sqrt{8})$ both features are present in our dynamical system.

Hence, thanks to Proposition 4.2 , when $2 \leq \mu<2+\sqrt{8}$ the invariant compact interval we need in the covering interval strategy is the absorbing interval $I=\left[\mathrm{m}^{\prime}, M^{\prime}\right]$. Moreover, for $\mu>4$ the natural choice for the covering intervals is given by

$$
\begin{aligned}
& I_{0}=\left[-1, \frac{-\mu-\sqrt{\mu^{2}-4 \mu}}{2 \mu}\right], I_{1}=\left[\frac{-\mu+\sqrt{\mu^{2}-4 \mu}}{2 \mu}, \frac{\mu-\sqrt{\mu^{2}-4 \mu}}{2 \mu}\right] \text { and } \\
& I_{2}=\left[\frac{\mu+\sqrt{\mu^{2}-4 \mu}}{2 \mu}, 1\right],
\end{aligned}
$$

whose extreme values are zeros of map $f$ or the solutions to the equation $|f(x)|=1$. Notice that $I_{0}, I_{1}$ and $I_{2}$ are the three intervals highlighted on the $x$-axis in Figure 6 . The corresponding transition matrix turns out to be

$$
\mathbf{T}_{f}=\left(\begin{array}{lll}
0 & 0 & 1 \\
1 & 1 & 1 \\
1 & 0 & 0
\end{array}\right)
$$

and a simple computation shows that it is not irreducible as

$$
\mathbf{T}_{f}^{n}=\left(\begin{array}{ccc}
0 & 0 & 1 \\
n & 1 & n \\
1 & 0 & 0
\end{array}\right)
$$

when $n$ is odd and

$$
\mathbf{T}_{f}^{n}=\left(\begin{array}{lll}
1 & 0 & 0 \\
n & 1 & n \\
0 & 0 & 1
\end{array}\right)
$$

when $n$ is even. Thus the covering interval approach does not allow to draw any conclusion on the existence of chaos, at least for the choice of the covering intervals above.

Somewhat similarly, also the SAP method seems to be not applicable in view of proving the presence of complex dynamics in our framework. In fact, given a compact interval $I$ on which map $\phi$ we are analysing is continuous, that technique in the $1 \mathrm{D}$ setting requires to find (at least) two disjoint compact sub-intervals $I_{0}$ and $I_{1}$ of $I$ such that their $\phi$ images cover $I$. In our context, however, taking $I=[-1,1]$, if $\mu<4$ then no such subintervals exist for map $f$, while if $\mu>4$, just one such sub-interval exists, which can be chosen in several ways. The smallest suitable one is $S_{2}$ in (4.3), the largest is $I$ itself; any interval in between works as well. However, as it is easy to check, it is not possible to find two disjoint compact intervals as required by the SAP method, no matter what the value of $\mu$ is. Notice indeed that the $f$ image of the set $S_{1}$ in (4.2) covers $I$, but $S_{1}$ is not an interval. 
This simple example illustrates that, when compared to the expander method in [7], the SAP technique requires in general stricter conditions in order to be applied. On the other hand, the latter allows to draw stronger conclusions from a dynamical viewpoint as, for instance, it ensures the existence of fixed points and, in fact, the periodic orbits are dense in a suitable invariant set. The technique in [7] guarantees instead neither the existence of fixed points (see Figure 1.5 in [15], which is inspired to the bulging horseshoe in Figure 4 in [7]), nor of periodic points of any period (cf. [8]). Nonetheless, as seen in Proposition 4.1, the method in [7] still allows to draw some interesting conclusions from a dynamical viewpoint. For a more detailed comparison between the covering relations in $[7,12]$, we refer the interested reader to [16].

\section{Conclusion and future directions}

In this paper we proposed a framework to analyse the dynamical process of decision and opinion formation of two economic homogeneous and boundedly rational agents that interact and learn from each other over time. The decisional process presented in our model is an adaptive adjustment mechanism in which an agent takes into account the difference between his/her own opinion and the opinion of the other agent. The smaller that difference, the larger the weight put on the comparison of the opinions. We also assumed that if the distance between the two opinions is larger than a given threshold, then there is no interaction and the agents do not change their opinions anymore.

Introducing an auxiliary variable describing the distance between the opinions, we obtained a 1D dynamical system for which we found a steady state in the origin, corresponding to the unanimity scenario, and a continuum of steady states related to the situation with absence of interaction. Analytically, we investigated the stability of the unanimity steady state, the first flip bifurcation through which it loses the stability and the double pitchfork bifurcation of the second iterate, as well as the presence of chaotic dynamics. Numerically, we showed the subsequent period-doubling bifurcations of the coexisting periodic attractors leading to two coexisting periodic attractors, which then merge into a unique attractor, when increasing the reactivity parameter.

Future research should focus, for instance, on the extension of the model to the framework of three interacting agents. In this case, however, it seems in general not possible to reduce the dimensionality of the problem by introducing some auxiliary variables and thus the analytical tractability of the model gets drastically reduced.

Another interesting extension would concern the context of heterogeneous agents: in such case it would still be possible to obtain a 1D model through a change of variable, but the resulting model would be heavier to analyse than that studied in the present paper.

We finally stress that models of the kind presented here are also suitable to be applied, for instance, in the context of financial asset markets with boundedly rational agents that ground their buying and selling decisions on fundamental values, which can be continuously updated on the basis of a comparative process among agents.

\section{Notes}

1. Email: ahmad.naimzada@unimib.it

2. In what follows, we will assume $\alpha>1 / 2$, so that $-(2 \beta /(1-2 \alpha))$ is a positive quantity and thus there is no need to reverse inequalities when passing from (2.4) to (2.5). In fact, as it is easy to see, the case with $\alpha \leq 1 / 2$ is not interesting from a dynamical viewpoint.

3. Notice that map $f$ is continuous on $\mathbb{R}$, it is $C^{1}$ on $\hat{\mathbb{R}}:=\mathbb{R} \backslash\{ \pm((\mu+1) / \mu)\}$ and $C^{2}$ on $\hat{\mathbb{R}} \backslash\{0\}$. However, we do not need to compute derivatives of order higher than one in the origin. 
As regards the fixed points $x= \pm((\mu+1) / \mu)$, we stress that the left partial derivative $(\partial f / \partial x)^{-}((\mu+1) / \mu ; \mu)=\mu+2>1$, while the right partial derivative $(\partial f / \partial x)^{+}((\mu+1) /$ $\mu ; \mu)=1$, and, symmetrically, $\left.(\partial f / \partial x)^{-}(-((\mu+1) / \mu)) ; \mu\right)=1$, while $(\partial f / \partial x)^{+}(-((\mu+1) /$ $\mu) ; \mu)=\mu+2>1$. For the fixed points $y \in\{ \pm x: x \in((\mu+1) / \mu,+\infty)\}$ it holds instead that $(\partial f / \partial x)(y ; \mu)=1$, for every $\mu$. Thus, as stated above, all fixed points but the origin are not locally asymptotically stable.

4. Simple calculations show indeed that $x=(\mu+1) / \mu$, i.e. one of the fixed points, is the only point of period two for $f$ on $(1,(\mu+1) / \mu]$, and similarly $x=-(\mu+1) / \mu$ is the only point of period two for $f$ on $[-((\mu+1) / \mu),-1)$. As already remarked, all points in $(-\infty,-((\mu+$ $1) / \mu)) \cup((\mu+1) / \mu, \infty)$ are fixed points, too.

5. We chose to illustrate a rotated version of the Smale horseshoe map, instead of the original one in [18], because in this way the connection with the 1D framework will be more evident.

6. Actually, in the definition of expander in [7], connectedness is not required and thus any subset of $E$ containing $S_{1} \cup S_{2}$ is an expander, too. For simplicity, however, hereinafter we will consider only connected expanders.

7. Notice that the extreme values for $S_{1}$ and $S_{2}$ depending on $\mu$ are found by solving the equations $\left.f(x)\right|_{[-1,0]}=1$ and $\left.f(x)\right|_{[0,1]}=-1$, respectively. Those values are well defined for $\mu>4$.

8. Indeed, for $\mu \geq 2$ it holds that $m, M \in I$.

\section{References}

[1] J. Auslander and J.A. Yorke, Interval maps, factors of maps, and chaos, Tôhoku Math. J. 32 (1980), pp. 177-188.

[2] M. Bianchi (ed.), The Active Consumer: Novelty and Surprise in Consumer Choice. Routledge, London, 1998.

[3] L. Block, J. Guckenheimer, M. Misiurewicz, and L.S. Young, Periodic points and topological entropy of one-dimensional maps, in Global Theory of Dynamical Systems, (Proc. Internat. Conf., Northwestern Univ., Evanston, Ill., 1979). Lecture Notes in Mathematics 819, 1980, pp. $18-34$.

[4] K. Burns and H. Weiss, A geometric criterion for positive topological entropy, Commun. Math. Phys. 172 (1995), pp. 95-118.

[5] P.M. DeMarzo, D. Vayanos, and J. Zwiebel, Persuasion bias, social influence and unidimensional opinions, Quart. J. Econ. 118 (2003), pp. 909-968.

[6] S.N. Elaydi, Discrete Chaos, Chapman \& Hall/CRC, Boca Raton, 2000.

[7] J. Kennedy, S. Koçak, and J.A. Yorke, A chaos lemma, Amer. Math. Monthly 108 (2001), pp. 411-423.

[8] J. Kennedy and J.A. Yorke, Topological horseshoes, Trans. Amer. Math. Soc. 353 (2001), pp. $2513-2530$.

[9] B.P. Kitchens, Symbolic Dynamics. One-Sided, Two-Sided and Countable State Markov Shifts, Universitext, Springer-Verlag, Berlin, 1998.

[10] T.Y. Li and J.A. Yorke, Period three implies chaos, Amer. Math. Monthly 82 (1975), pp. $985-992$.

[11] D. Lind and B. Marcus, An Introduction to Symbolic Dynamics and Coding, Cambridge University Press, Cambridge, 1995.

[12] A. Medio, M. Pireddu, and F. Zanolin, Chaotic dynamics for maps in one and two dimensions. A geometrical method and applications to economics, Int. J. Bifur. Chaos Appl. Sci. Eng. 19 (2009), pp. 3283-3309.

[13] K. Mischaikow and M. Mrozek, Isolating neighborhoods and chaos, Japan J. Indus. Appl. Math. 12 (1995), pp. 205-236.

[14] A. Naimzada and M. Pireddu, Adaptive decision dynamics: Bifurcations, multistability and chaos, Appl. Math. Comput., forthcoming.

[15] M. Pireddu, Fixed points and chaotic dynamics for expansive-contractive maps in Euclidean spaces, with some applications, Ph.D. thesis, University of Udine, Italy, 2009. Available at http://arxiv.org/abs/0910.3832

[16] M. Pireddu and F. Zanolin, Some remarks on fixed points for maps which are expansive along one direction, Rend. Istit. Mat. Univ. Trieste 39 (2007), pp. 245-274.

[17] M. Pireddu and F. Zanolin, Chaotic dynamics in the Volterra predator-prey model via linked twist maps, Opuscula Math. 28(4) (2008), pp. 567-592. 
[18] S. Smale, Diffeomorphisms with many periodic points, in Differential and Combinatorial Topology (A Symposium in Honor of Marston Morse) Princeton University Press, Princeton, NJ, 1965, pp. 63-80.

[19] A. Szymczak, The Conley index and symbolic dynamics, Topology 35 (1996), pp. 287-299.

[20] P. Walters, An Introduction to Ergodic Theory, Graduate Texts in Mathematics Vol 79, Springer-Verlag, New York, 1982.

[21] S. Wiggins, Introduction to Applied Nonlinear Dynamical Systems and Chaos, Texts in Applied Mathematics, Springer-Verlag, New York, 1990.

[22] P. Zgliczyński and M. Gidea, Covering relations for multidimensional dynamical systems, J. Differ. Equ. 202 (2004), pp. 32-58. 